Kliebe, Lena

\title{
Hochschullernwerkstatt als Raum und pädagogische Konzeption - "Mein eigener Lernprozess"
}

Kramer, Kathrin [Hrsg.]; Rumpf, Dietlinde [Hrsg.]; Schöps, Miriam [Hrsg.]; Winter, Stephanie [Hrsg.]: Hochschullernwerkstätten - Elemente von Hochschulentwicklung? Ein Rückblick auf 15 Jahre Hochschullernwerkstatt in Halle und andernorts. Bad Heilbrunn : Verlag Julius Klinkhardt 2020, S. 157-166. - (Lernen und Studieren in Lernwerkstätten)

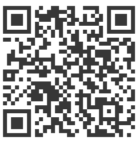

Quellenangabe/ Reference:

Kliebe, Lena: Hochschullernwerkstatt als Raum und pädagogische Konzeption - "Mein eigener Lernprozess" - In: Kramer, Kathrin [Hrsg.]; Rumpf, Dietlinde [Hrsg.]; Schöps, Miriam [Hrsg.]; Winter, Stephanie [Hrsg.]: Hochschullernwerkstätten - Elemente von Hochschulentwicklung? Ein Rückblick auf 15 Jahre Hochschullernwerkstatt in Halle und andernorts. Bad Heilbrunn : Verlag Julius Klinkhardt 2020, S. 157-166 - URN: urn:nbn:de:0111-pedocs-212090 - DOI: 10.25656/01:21209

https://nbn-resolving.org/urn:nbn:de:0111-pedocs-212090

https://doi.org/10.25656/01:21209

in Kooperation mit / in cooperation with:

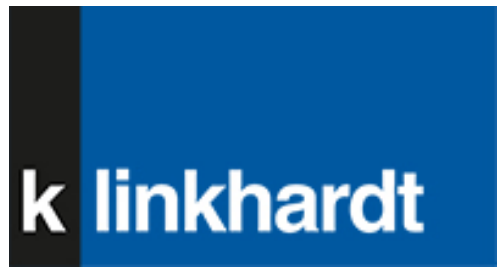

http://www.klinkhardt.de

\section{Nutzungsbedingungen}

Dieses Dokument steht unter folgender Creative Commons-Lizenz: http://creativecommons.org/licenses/by-nc-sa/4.0/deed.de - Sie dürfen das Werk bzw. den Inhalt unter folgenden Bedingungen vervielfältigen, verbreiten und öffentlich zugänglich machen sowie Abwandlungen und Bearbeitungen des Werkes bzw. Inhaltes anfertigen: Sie müssen den Namen des Autors/Rechteinhabers in der von ihm festgelegten Weise nennen. Dieses Wutork bzw. der Inhalt darf nicht für kommerzielle Zwecke verwendet werden. Die neu entstandenen Werke bzw. Inhalte dürfen nur unter Verwendung von Lizenzbedingungen weitergegeben werden, die mit denen dieses Lizenzvertrages identisch oder vergleichbar sind.

Mit der Verwendung dieses Dokuments erkennen Sie die Nutzungsbedingungen an.

\section{Terms of use}

This document is published under following Creative Commons-License: http://creativecommons.org/licenses/by-nc-sa/4.0/deed.en - You may copy, distribute and transmit, adapt or exhibit the work in the public and alter, transform or change this work as long as you attribute the work in the manner specified by the author or licensor. You are not allowed to make commercial use of the work. If you alter, transform, or change this work in any way, you may distribute the resulting work only under this or a comparable license.

By using this particular document, you accept the above-stated conditions of use.

\section{Kontakt / Contact:}

pedocs

DIPF | Leibniz-Institut für Bildungsforschung und Bildungsinformation

Informationszentrum (IZ) Bildung

E-Mail: pedocs@dipf.de

Internet: www.pedocs.de

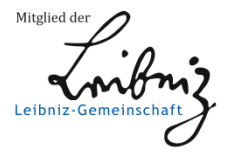




\section{Lernen und Studieren in Lernwerkstätten}

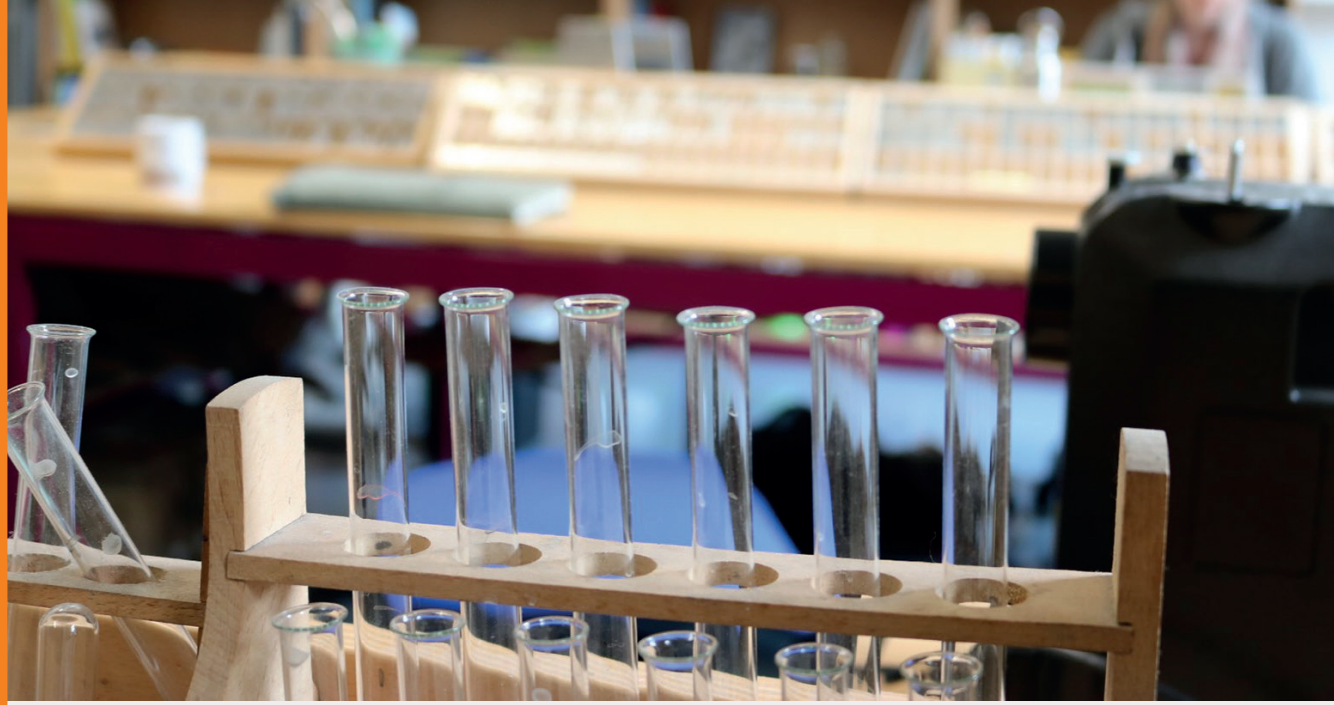

Kathrin Kramer / Dietlinde Rumpf / Miriam Schöps / Stephanie Winter (Hrsg.)

Hochschullernwerkstätten - Elemente von Hochschulentwicklung?

Ein Rückblick auf 15 Jahre Hochschullernwerkstatt in Halle und andernorts 


\section{Kramer / Rumpf / Schöps / Winter Hochschullernwerkstätten - Elemente von Hochschulentwicklung?}




\section{Lernen und Studieren in Lernwerkstätten}

Impulse für Theorie und Praxis

Herausgegeben von

Eva-Kristina Franz, Johannes Gunzenreiner, Barbara Müller-Naendrup, Hartmut Wedekind und Markus Peschel 


\section{Kathrin Kramer \\ Dietlinde Rumpf \\ Miriam Schöps \\ Stephanie Winter \\ (Hrsg.)}

\section{Hochschullernwerkstätten - Elemente von Hochschulentwicklung?}

Ein Rückblick auf 15 Jahre Hochschullernwerkstatt in Halle und andernorts 
Der vorliegende Band ist anlässlich einer Tagung des 15jährigen Bestehens der Hochschullernwerkstatt Erziehungswissenschaften in Halle im November 2020 entstanden.

Gefördert wurden die Tagung und der Band vom Zentrum für Lehrer *innenbildung, der Universitäts- und Landesbibliothek Sachsen-Anhalt sowie der Martin-Luther-Universität Halle-Wittenberg.

Dieser Titel wurde in das Programm des Verlages mittels eines Peer-Review-Verfahrens aufgenommen. Für weitere Informationen siehe www.klinkhardt.de.

Bibliografische Information der Deutschen Nationalbibliothek Die Deutsche Nationalbibliothek verzeichnet diese Publikation in der Deutschen Nationalbibliografie; detaillierte bibliografische Daten sind im Internet abrufbar über http://dnb.d-nb.de.

2020.k. (C) by Julius Klinkhardt.

Coverfoto: (C) Florian Johnke-Liese / Hochschullernwerkstatt.

Druck und Bindung: AZ Druck und Datentechnik, Kempten.

Printed in Germany 2020.

Gedruckt auf chlorfrei gebleichtem alterungsbeständigem Papier.

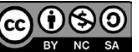

Die Publikation (mit Ausnahme aller Fotos, Grafiken und Abbildungen) ist veröffentlicht unter der Creative Commons-Lizenz: CC BY-NC-SA 4.0 International https://creativecommons.org/licenses/by-nc-sa/4.0/ 


\section{Inhalt}

Kathrin Kramer, Dietlinde Rumpf, Miriam Schöps und Stephanie Winter Einführung in den Band

\section{Teil 1: Historische Entwicklung}

Miriam Schöps und Dietlinde Rumpf

Universität mit Lernwerkstatt in Entwicklung -

Von der Materialausleihe zur Hochschuldidaktik

Hartmut Wenzel

Lernwerkstätten auch für die Sekundarstufen -

Zur Entwicklung der Hochschullernwerkstatt in Halle

Ulrike Stadler-Altmann, Susanne Schumacher, Enrico A. Emili,

Gerda Winkler und Elisabeth Dalla Torre

Hochschullernwerkstätten als Spielball der Bildungspolitik?

Die EduSpace Lernwerkstatt in der Südtiroler Lehrer*innenbildung zwischen nationalen und regionalen Bildungsinteressen

Hartmut Wedekind und Corinna Schmude

Von der Idee zum strukturell verankerten hochschuldidaktischen Prinzip -

Lernwerkstätten und Lernwerkstattarbeit im Studiengang „Erziehung und Bildung in der Kindheit" an der Alice Salomon Hochschule Berlin 68

\section{Teil 2: Wahrnehmung, Selbstverständnis, Einflüsse}

Dietlinde Rumpf und Corinna Schmude

NeHle - Internationales Netzwerk der Hochschullernwerkstätten -

Entwicklungsphasen einer Interessenvertretung und

eines gemeinsamen Begriffsverständnisses

Constantin Beyer und Florian Johnke-Liese

Hochschullernwerkstatt goes digital!

Alexandra und Michael Ritter

Drucken wie ,von gestern' in der Schule der Zukunft -

Die Schuldruckerei als Erfahrungsraum für Kinder und Studierende 
Livia Enders (geb. Makrinus) und Stephanie Winter

Studierende und Dozierende im Spannungsfeld zwischen

Theorie und Praxis - Die Hochschullernwerkstatt als Forschungsraum

John Marcus Sommer

Selbstwahrnehmung in der Hochschullernwerkstatt -

Wissenschaft zwischen Abstraktion und emotionalem Lernen

Lena Kliebe

Hochschullernwerkstatt als Raum und pädagogische Konzeption -

„Mein eigener Lernprozess“

Jerome Kampe

Geschichtsunterricht in der Lernwerkstatt!?

\section{Teil 3: Partizipation und Inklusion}

Melanie Schlag

Hochschullernwerkstatt schlägt Schlager

Kathrin Kramer

Nichtreformistische Reformen - Die Bedeutung von

Hochschullernwerkstätten auf dem Weg zu einer inklusiven Universität am Beispiel des Budgets für Arbeit

Johanna Ingenerf und Pascal Kurz

Menschenrechtsbasierte Bildung im internationalen Dialog -

Ein selbstorganisiertes Seminar mit Austausch

Ines Boban und Andreas Hinz

Hochschullernwerkstatt Halle als Möglichkeitsort -

Von WIRkstatt-Impulsen für inklusive Prozesse

Brigitte Kottmann und Alena Lensker (geb. Beckmann)

Die Lernwerkstatt und der Studiengang „Integrierte Sonderpädagogik“

an der Universität Bielefeld: Impulse, Synergien und Reflexionen

Sarah Dannemann, Tjark Neugebauer, Claudia Schomaker und Rolf Werning

Die LeibnizLernlandschaft: Diversität und Digitalisierung $\left(\mathrm{L}^{2} \mathrm{D}^{2}\right)$

gestalten - Konzeptionelle Gedanken für eine inklusive

Hochschullernwerkstatt an der Leibniz Universität Hannover 


\section{Teil 4: Reflexivität und Professionalisierung}

Kira Wybierek

Praxissemester in der Hochschullernwerkstatt -

Reflexion eines Lernprozesses

Eva Hoffart

„...da man lernt, eigene Gedanken und Ideen noch einmal zu vertiefen“ -

Theoretische Überlegungen und praktische Umsetzungen zum Reflektieren

von Lehramtsstudierenden

Edita Jung und Lena S. Kaiser

„Vielleicht romantisiere ich die Räume meiner Kindheit.“

Zugänge zu einer biographischen Reflexivität in

kindheitspädagogischen Hochschullernwerkstätten

Rolf-Torsten Kramer

Zum Problem der Professionalisierung im Lehramtsstudium und

zum Potenzial der Hochschullernwerkstatt

Mark Weißhaupt, Kathleen Panitz und Elke Hildebrandt

Die Inszenierung von „Theorie und Praxis“ sowie

„Neugier und Desinteresse“ bei der Professionalisierung

angehender Lehrpersonen in Hochschullernwerkstätten

Markus Peschel und Pascal Kihm

Hochschullernwerkstätten - Rollen, Rollenverständnisse und

Rollenaushandlungen

David Paulus, Patrick Gollub und Marcel Veber

Forschendes Lernen und Kasuistik: Überschneidungen und

Abgrenzungen bezogen auf Reflexivität in der

Hochschullernwerkstattarbeit

Pascal Kihm, Jenny Diener und Markus Peschel

Qualifizierungsprozesse und Qualifikationsarbeiten in

Hochschullernwerkstätten - Forschende Entwicklung einer

innovativen Didaktik 
10| Inhaltsverzeichnis

\section{Teil 5: Kooperation}

Georg Breidenstein, Sara Burkhardt, Thorid Rabe und Miriam Schöps

Zur Materialität des Lernens -

Anregungen aus einem interdisziplinären Forum

in der Hochschullernwerkstatt

Miriam Asmus, Kati Friebe, Mirjam Lewin und Kati Misselwitz

Entdeckendes Lernen und Digitale Medien - Ein Traumpaar .

Siglinde Spuller

Kooperation und Kooperatives Lernen als Prinzip

Hochschullernwerkstätten-adäquaten Lernens?

Eine konzeptionelle Verortung

Linda Balzer

Der Effekt selbstregulierenden und forschenden Lernens auf kooperative und individuelle Lernprozesse in der

Lernwerkstatt Religion Plural (LeRP)

Martin Lindner

Werkstattarbeit in der Biologiedidaktik -

Umstellung eines Praktikums auf Projektarbeit

Autor*innenbeschreibungen 
Lena Kliebe

\section{Hochschullernwerkstatt als Raum und pädagogische Konzeption - „Mein eigener Lernprozess“}

\section{Zusammenfassung}

Die Hochschullernwerkstatt der Martin-Luther-Universität Halle-Wittenberg bietet den Studierenden innerhalb eines Semesters unter verschiedenen Schwerpunkten Seminare und Workshops an. Unsere Seminargruppe lernte im Rahmen des Moduls "Schulgeschichte und Schulgestaltung" die Lernwerkstatt als Raum und pädagogische Konzeption kennen. Unter der Leitung von Dr. Dietlinde Rumpf und Kathrin Kramer konnte man direkte Einblicke in die Arbeit von Lernbegleiter*innen gewinnen und selbst die Rolle von Lernenden übernehmen. Dadurch erhielten wir eine der wenigen Möglichkeiten für Student*innen im Hochschulstudium, bewusst den eigenen Lernprozess wahrzunehmen und zu reflektieren. Die von mir gesammelten Erfahrungen, Einblicke und Erkenntnisse werden im Folgenden beschrieben und reflektiert. Dadurch werden Transferüberlegungen in den schulischen Kontext hergestellt, die mögliche Handlungsweisen beziehungsweise den Umgang mit den gewonnenen Kenntnissen in der Schule aufzeigen sollen.

\section{Wie habe ich die Lernsituation und das Arrangement erlebt?}

Durch mein bestehendes Arbeitsverhältnis in der Hochschullernwerkstatt Erziehungswissenschaften in Halle (als studentische Mitarbeiterin) erlebe ich diese schon lange als einen Ort der Begegnung, des Austauschs, der kreativen Prozesse und Lernerfahrung, gleich ob extrinsisch oder intrinsisch motivierten Ursprungs. Bisher habe ich diese Prozesse eher passiv und meist nur oberflächlich beobachten können, weshalb mich das Konzept dieses Seminars sehr reizte.

Das in diesem Rahmen vorgestellte Seminar (innerhalb des Moduls „Schulgeschichte und Schulgestaltung") trägt den Titel „Die Lernwerkstatt als Raum und als pädagogische Konzeption" und konnte von Lehramtsstudierenden jeder Schulform belegt werden. Insgesamt gab es vier Arbeitsphasen: Theorieinput, Fragenformulierung, Erarbeitungsphase und Reflexion. Hierbei erhielten die Studierenden die Chance mit selbstentwickelten Fragestellungen die Arbeitsweise als Lernende 
oder auch als Lernbegleiter*innen in einer Hochschullernwerkstatt praktisch zu erleben und zu begreifen. Dadurch konnten gewonnene Erkenntnisse (beispielsweise in den Bereichen: Bewusstwerden der eigenen Lernprozesse, Umgang mit dem problemorientierten-induktiven Lösen von Aufgaben und selbstbestimmtes/ selbstorganisiertes und selbstverantwortetes Arbeiten) in Hinblick auf die eigene Professionalisierung zukünftiger Schulgestaltung und schulisches Lernen kritisch reflektiert werden.

Es ebnete mir den Weg, die Lernwerkstatt(arbeit) aus einem differenzierteren Blickwinkel zu betrachten. So erhielt ich die Chance, aktiv mein eigenes Lernen und das der anderen in der Hochschullernwerkstatt beobachten, erleben und analysieren zu können. Das eigene Lernen und die zur Verfügung gestellte Lernumgebung nicht nur im Nachhinein, sondern auch während des Prozesses aktiv zu betrachten, sind doch eher ungewöhnlich. Man beginnt nicht nur ergebnisorientiert zu denken, sondern konzentriert sich verstärkt auf den eigenen Weg zum Ergebnis und auf die Prozesse der anderen. Es herrschte insgesamt eine sehr lockere und offene Lernatmosphäre, die vermutlich nicht nur durch die selbstbestimmende Lernsituation, die unterstützenden Lernbegleiter*innen, sondern auch durch den besonderen Lernort entstand.

Die drei Räume der Hochschullernwerkstatt besitzen offene Regale, die impulsgebende Materialien beinhalten, welche inhaltlich nach verschiedenen Themen strukturiert sind und Lebensbezug aufweisen. Zudem findet man Rückzugsmöglichkeiten, wie die Sofaecken, oder nutzt die Räume als einen Ort der Begegnung, um beispielsweise gemeinsame Ziele abzustecken oder um kreativ zu arbeiten wie in der (Druck-)Werkstatt. Dabei erzeugen die drei Räume generell unterschiedliche Gefühle in mir, was sich in diesem Seminar auf meine Arbeitsweise übertrug. Der „Montessori-Raum“ wirkt im Vergleich zum Werkstattraum zwar einladend, aber mehr wie ein offener Klassenraum auf mich. Die Tische und Stühle ähneln in Form und Farbe Schulbänken, auch Wandregale gefüllt mit Büchern findet man in Klassenräumen und auch die Computerecke könnte es so in jenen geben. Durch die Pflanzen, die Teppiche und die gelben Sofas, die als Sitzecken dienen, erhält der Raum allgemein einen freundlichen, einladenden und gemütlichen Touch. Dennoch fühlte ich mich in der Zeit des Seminars zur Atmosphäre des „Werkstattraums“ am meisten hingezogen, weswegen ich alle eigenständigen Lernphasen dort verbracht habe. Ich kann nicht genau beschreiben, weshalb mich das handwerkliche Arrangement in dem Raum so fesselt, aber ich kann vermuten, dass mich für die Seminarzeit die (kleine) Größe des Raumes und die vielen Geräte (wie Werkbank, Druckerpresse etc.) mehr zum kreativen Nachdenken anregten, als ein großer offener Raum (wie zum Beispiel der „Bühnenraum“). So ist es doch interessant zu erwähnen, dass ich, trotz der gemütlichen Sofas im „MontessoriRaum“, nicht einmal in Erwägung gezogen hatte, den Raum zu wechseln. Ein Grund könnte meine Affinität zum bildnerischen Arbeiten und zum Buchbin- 
den sein, weshalb mich allgemein die Atmosphäre einer Werkstatt entspannt und kreativ anregt. So lässt sich vielleicht erklären, warum mich die Größe und die „Leere" des „Bühnenraums" nicht ansprachen - ich habe den geringsten Bezug zu ihm. Zwei weitere Faktoren sollten noch beleuchtet werden. Einerseits benötigte ich für mein eher persönliches Thema (Warum tue ich mir Semester für Semester das Pendeln an?) keine Fachliteratur und konnte daher raumunabhängig agieren. Andererseits konnten sich in der Werkstatt viele Gespräche entwickeln, die mitunter zwei bis vier Leute betrafen, ohne, dass man die Lautstärke verändern musste. Dass man in diesem Raum oft dazu verleitet wurde Gespräche zu führen oder sich gegenseitig zu helfen, kann auch an der gruppenähnlichen (aufgrund der Raumgröße und Blickrichtung mit der wir saßen/standen) Sitzkonstellation liegen, die für das Führen von Gesprächen prädestiniert ist (vgl. Woolfolk, 2014, 444).

Ein großer Faktor, der mich in meinem Lernprozess überraschte, war meine Offenheit gegenüber Gesprächen mit den anderen Seminarteilnehmer*innen und den daraus gewonnenen Kenntnissen, worauf ich im Folgenden näher eingehen werde.

\section{Wie habe ich die Gruppe wahrgenommen?}

Trotz anfänglicher Distanziertheit, wie man sie häufig in der ersten Phase eines neuen Seminars vorfindet, gewann man in der Kennlernrunde einen ersten Eindruck der Teilnehmer*innen, was die Anspannung etwas auflockerte. Nach der Vorstellung des Seminarthemas („Die Lernwerkstatt als Raum und pädagogische Konzeption“) und der Aufgabe („Übernehmen Sie die Rolle der lernenden Person (eigene Fragestellung entwickeln) oder die der Lernbegleitung (den Lernprozess der anderen begleiten) und reflektieren Sie Ihre Lernerfahrung in Hinblick auf schulisches Lernen“), konnte ich anfangs bei den Teilnehmer*innen eine große Verwirrung bezüglich der Aufgabenstellung und der Zielführung des Seminars spüren. Auch trafen die selbstständige Themenwahl und die Reflexion des eigenen Lernprozesses auf großen Unmut bei einigen Teilnehmer*innen, was sie untereinander leise verbal äußerten. Dabei liegt eine Vermutung von mir darin, dass einige von ihnen bisher kaum mit Hochschullernwerkstattarbeit in Berührung kamen und dass der praktische, individuelle Weg der selbstständigen Erarbeitung eines neuen Themas eher untypisch für die ihnen bisher bekannte, angeleitete Hochschulwissensaneignung war. Darüber hinaus kam es in meinem Studium selten vor, dass Studierende von ihren Dozierenden in ihren Lernprozessen begleitet wurden oder dass sie sogar selbst in die begleitende Rolle schlüpfen durften. Und wenn das schon nicht genug gewesen wäre, das anschließende Analysieren und Reflektieren darüber kam noch seltener vor. So kann es auch meinen 
Kommiliton*innen ergangen sein, weshalb bei ihnen ebenfalls eine Unsicherheit entstanden sein könnte. Nach Pietsch (2010) könnte der Grund der mangelnden Umsetzung daran liegen, dass die Studierendenzahl in Lehramtsstudiengängen zu hoch ist, um schlicht und ergreifend ein solches Konzept möglichst weitgreifend durchzuführen (vgl. ebd., 226).

Diese Umstellung zum Selbstmanagement verlangte eine gewisse Zeit. Dass die Entwicklung von Selbstmanagement und Verantwortungsübernahme zeitaufwendig und notwendig für kooperative und problemorientierte Lernaufgaben ist, bestätigt auch Anita Woolfolk $(2014,473)$. Doch von Seminareinheit zu Seminareinheit, insbesondere jedoch durch das gemeinsame Wochenende (neben einigen Einzelseminaren in der Woche, gab es auch ein gemeinsames Blockwochenende, in dem wir speziell an der Bearbeitung/Lösung unserer Leitfrage arbeiteten), haben fast alle einen Weg gefunden, mit der neuen Lernsituation umzugehen und sich für ein eigenes Thema oder für die Rollenübernahme einer*eines Lernbegleiterin*Lernbegleiters zu entscheiden. Dabei haben sich von den etwa zwei Dutzend Teilnehmenden allerdings nur zwei für die Rolle der Lernbegleitung entschieden, alle anderen wählten die der*des Lernenden. Die beiden Dozentinnen wählten ebenfalls die Rolle der Lernbegleitung.

Im Laufe der Seminareinheiten baute ich zu der einen oder anderen Person ein sehr offenes und fast freundschaftliches Verhältnis auf. Ich kam mit ihnen sehr oft ins Gespräch, welches teilweise von allgemeinen bis sehr persönlichen Themen reichte. Da ich selber ein sehr persönliches Thema wählte, musste ich mich recht schnell den anderen Teilnehmer*innen öffnen, was mir zwar nicht ganz leicht fiel, mir aber in meinem Prozess weiterhalf. Ich konnte durch den Austausch von Ratschlägen, Erfahrungen, Einstellungen, Vermutungen und Analysen mehr über mich selbst erfahren, was mich erstaunte. Ging ich doch davon aus, alleine arbeiten zu müssen, da ja nur ich am besten über mich Bescheid wisse.

Es gab aber auch viele Momente, in denen ich einfach den anderen bei ihren Gesprächen zuhörte, da mich diese ebenfalls interessierten und ich eventuell mit Rat zur Seite stehen konnte. So erinnere ich mich besonders an das Gespräch mit einem Lernbegleiter und einer Lernenden, bei dem es sich um die Tag- und Nachtdefinition handelte. Es entstand eine Diskussion, die subjektive und objektiv-wissenschaftliche Definitionen gegeneinander abwog. Dabei empfand ich die konträren Sichtweisen als sehr spannungsvoll. Die beiden Diskutierenden schlugen immer wieder neue Gedankengänge ein und bereicherten so den Argumentationswechsel. Sie schätzten die Meinung des*der jeweils anderen wert, indem sie sie nicht verneinten, sondern mit den eigenen Überzeugungen/Argumenten oder Sichtweisen verglichen, und neue Rückschlüsse zuließen. Auch wenn ich nichts explizit zu dem Gespräch beisteuerte, so regte es doch eine eigene Hinterfragung der Tag-Nachtdefinition bei mir an, weswegen ich mich in diesem Kontext nicht nur als Zuhörerin betrachtete, sondern auch als Lernende. Insgesamt wirkten viele 
Teilnehmer*innen auf mich den Themen anderer gegenüber sehr aufgeschlossen, was den Eindruck eines freundlichen und empathischen Umgangs miteinander erweckte. Es gab zwar vereinzelt Leute, die alleine ihren Prozess erleben wollten, doch wirkten sie (auf mich) von der Gruppe nicht ausgeschlossen. Auch wenn es Weitere gab, die lange Zeit nicht wussten, was ihr Thema ist oder wie sie weiter verfahren sollten, hatte ich das Gefühl, dass sie durch die Gespräche untereinander neue Ideen, Erkenntnisse oder Mut fanden, ihr Thema weiterzuverfolgen oder abzulegen. So konnte sich durchaus die Tür für eine neue Strategie oder ein neues selbstgewähltes Thema öffnen.

\section{Wie habe ich die Lernbegleitung wahrgenommen?}

Uns standen in der Erarbeitungsphase aktiv vier Lernbegleiter*innen zur Seite, die uns entweder direkt ansprachen oder selbst angesprochen werden konnten. Man selber musste anfangs entscheiden, ob man eher eine Lernbegleiter*innenrolle übernehmen will oder lieber einen eigenen Lernprozess durchlaufen möchte. Ich entschied mich gegen die Rolle der Lernbegleiterin, da ich mich verstärkt auf den eigenen Lernprozess konzentrieren und mich aktiv mit ihm auseinandersetzen wollte. Dabei wählte ich ganz bewusst diese Rolle, weil ich mein Lernen optimieren wollte und um das zu erreichen, musste ich mich mit meinem persönlichen Lernprozess aktiv auseinandersetzen.

Ich empfand die Anwesenheit der Lernbegleiter*innen als sehr angenehm und keineswegs aufdringlich. Sie waren Gesprächspartner*innen, die mir zugehört und mich entweder durch Fragen und Schilderungen eigener Erfahrungen im Arbeitsprozess weitergebracht oder zum Umdenken bewegt hatten. Dies erwies sich an manchen Stellen als sehr nützlich. So zum Beispiel das Gespräch mit einem Lernbegleiter, der mir aufzeigte, dass ich zu sehr in die Zukunft denke und zu wenig im Hier und Jetzt. Dieser Schritt erwies sich als sehr hilfreich, da sich die Stagnation des Prozesses auflöste und ich neu ansetzen konnte.

Aber nicht nur die Lernbegleiter*innen, die sich aktiv in diese Rolle begaben, wurden ihren Aufgaben gerecht. Meiner Meinung nach sollte die Lernbegleitung den*die Lernende* $n$ beim Lernprozess unterstützen, indem sie beratend zur Seite steht und das auf Augenhöhe. Dabei stellt die Lernbegleitung keine zwingend wissende Instanz dar. Somit kann sie auch lediglich als Zuhörer*in dienen und/oder sich gemeinsam mit der lernenden Person auf die Problemlösung einlassen. Auch die anderen Teilnehmer*innen nahmen stellenweise die Rolle der Lernbegleiter*innen ein. So unterstützten sie unbewusst oder vielleicht auch ganz bewusst die Lernprozesse der anderen durch Gespräche, was sie in gemeinsamen Auswertungsrunden anmerkten. Dies konnte ich ebenfalls in meinem Prozess erkennen. Denn durch die anregenden Denkanstöße und Fragen der anderen 
Seminarteilnehmer*innen, wurde mein Prozess maßgeblich angetrieben. Ganz besonders erinnere ich mich dabei an das Gespräch mit A., was mich sehr aufwühlte. Es war ein sehr persönliches Gespräch, in dem Ängste, Bedenken oder eigene Erfahrungen geäußert wurden. Es hat zwar meinen Lerninhalt zu weit in die Zukunft gerückt, doch setzte ich mich verstärkt mit meinen Gefühlen und Bedürfnissen auseinander, die entscheidend für die Klärung meiner Fragestellung waren. Aber nicht nur die anderen begaben sich ab und an in die Lerngebegleiter*innenrolle, sondern auch ich selber befand mich zwischendrin in einer Phase der Lernbegleitung, indem ich die Vorgänge der anderen hinterfragte oder sie mit meinen Gedanken zum Fortschreiten ihres Lernprozesses anregte. Beispielsweise offenbarte ich A. meine persönliche Sichtweise zu ihrem Thema „Ästhetik“, die sich von ihrem Verständnis zu dem Begriff „Ästhetik“ unterschied. Dadurch geriet sie ins Umdenken und hinterfragte diesen Begriff auf eine neue Art.

Letztlich kann ich daher feststellen, dass man auf verschiedenen Wegen die Lernbegleitung wahrnehmen kann. Egal ob bewusst oder unbewusst, sie ist in jedem Fall für mich von großer Bedeutung gewesen und hat meinen Prozess maßgeblich bestimmt.

\section{Transferüberlegungen: Eigenes Thema - Schulbezug}

Die Wahl meines Themas war schnell getroffen, da mich jenes zu dieser Zeit sehr beschäftigte. Es lautete: „Warum tu ich mir Semester für Semester das Pendeln an?““. Es ist ein sehr persönliches Thema, was sich schlecht irgendwo nachforschen lässt. Beziehungsweise musste ich, um Antworten zu finden, tief in meine Gefühle, Gedanken und Bedürfnisse eintauchen. Daher nahm ich an, dass ich vor allem alleine arbeiten müsse, da ja nur ich am besten über mich Bescheid wisse. Doch es stellte sich schnell heraus, dass mir das Sprechen mit anderen weitaus mehr half, als alleine in meinen Gedanken zu verweilen. Sie gaben mir viele Anregungen zum Nachdenken, wie zum Beispiel eine App zu nutzen, die meinen Tagesablauf dokumentiert, den Schritt zu wagen nach Halle zu ziehen, die S-Bahnfahrt lieber effektiv für das Studium zu nutzen, Einteilung meiner Termine in flexibel oder statisch, meinen Alltag besser durchzustrukturieren oder ganz einfach auch einmal zu akzeptieren „faul“ zu sein. Es ist ein sehr subjektiver Arbeitsprozess, welcher Gefahr läuft, den Fokus zu verlieren.

Durch die hohe Anzahl an Gesprächen, nicht nur zu meinem Thema, verlor ich daher manchmal meine eigene Fragestellung aus den Augen und habe mich mehr den anderen Themen gewidmet. Daher sollte man sich immer wieder bewusst werden, in welcher Rolle man sich eigentlich befindet - die der Lernenden oder die der*des Begleiterin*Begleiters. 
Letztlich habe ich das Ende der Erarbeitungsphase dafür genutzt, mich nochmals bewusst allein mit dem Thema auseinanderzusetzen. Ich fokussierte mich nun auf die Pendelzeit, da ich feststellen konnte, dass mich diese am meisten belastete und das Thema „Umzug nach Halle“ zu zukunftsorientiert war. Ich kam zu dem Schluss, dass ich alleine bereits am Montag schon 53 min einsparen könnte, wenn ich mit dem Fahrrad fahren würde. Weshalb ich mir die Fragen stellte, ob ich Fahrradfahren in Erwägung ziehen sollte: Ist das das Problem? Auch versuchte ich mir eine eigene Pausendefinition herzuleiten, um das eigene „Faulsein“ zu legitimieren. Ich verließ schließlich das Seminar mit gemischten Gefühlen und konnte die Fragen zu diesem Zeitpunkt nicht für mich beantworten. Allerdings gossen mir die persönlichen Sichtweisen der anderen Teilnehmenden und die aktive Auseinandersetzung mit dem Thema ein gutes Fundament, um mich weiterhin damit auseinanderzusetzen. Bis heute prägt mich diese Zeit, da ich immer wieder an die Gespräche mit den Lernenden und den*der Lernbegleiter*innen denken muss und mein Handeln und Denken immer wieder auf's Neue reflektiere.

In Bezug auf Schule können sich „Zeitstrukturierung im Alltag“ oder ganz allgemein das Thema "Zeit“ im Rahmen von Lernwerkstattarbeit eignen. Hierbei könnten sich die Schüler*innen ganz individuell dem Thema nähern. Das macht dieses offene Konzept so spannungsvoll - der Einfluss der unterschiedlichen Herangehensweisen und Perspektiven kann ein doch schlicht erscheinendes Thema, später so divers und vielfältig erscheinen lassen, wie man es vorher nicht vermutet hätte (beispielsweise die Nacht-Dunkeldefinition). Wiederum kann ich mir sehr gut vorstellen, dass die Schule mithilfe von Lernwerkstattarbeit auch individuelleren und letztlich persönlicheren Themen Raum geben sollte. In Form von gemeinsam erarbeiteten Kriterien könnte den Kindern beim Reflektieren ihres Prozesses und Ergebnisses geholfen werden. Sie würden dabei nicht nur mehr über sich selbst lernen, mit ihrem Interessenhorizont arbeiten und ein Selbstkonzept entwickeln, sondern auch den Prozess des selbstständigen/selbstbestimmenden Lernens erleben.

Ich entnehme aus der im Seminar erlebten Lernwerkstattarbeit eine spannende Zeit, die mir unter anderem viel Aufschluss über mein eigenes Lernen gab. So schrieb John Dewey $(2011,206)$, dass man dem Lerngegenstand am besten so begegnen würde, wie wir es auch außerschulisch im Alltag machen würden. Denn so kann das absichtliche Beobachten von Beziehungen einen ungeahnten Lernprozess in Gang setzen. Ich bin dem Thema durch Gespräche näher gekommen, wie ich es auch in außerschulischen, in alltäglichen Situationen tun würde. Mir halfen diese Gespräche, mehr über mich und mein Lernverhalten zu erfahren. Es hat sich herausgestellt, dass ich unter anderem eine kommunikative Lernerin bin (was mir vorher nicht so bewusst war). Ich konnte beobachten, dass auch ein großer Teil der anderen Teilnehmer*innen sehr viele Gespräche geführt hat und ihnen diese in ihrem Prozess weiterhalfen beziehungsweise sich als unabdingbar 
für ihr Vorankommen herausstellten. Daher nehme ich an, dass dies ein wichtiger Aspekt in meiner künftigen Schularbeit werden könnte - die Schüler*innen mehr miteinander ins Gespräch kommen zu lassen. Durch das Prinzip der Lernwerkstattarbeit wurden diese geradezu angekurbelt. Der Unterschied jedoch von „ImAlltag-Lernen“ $\mathrm{zu}$ „Lernen in der Schule“ oder „Lernwerkstattarbeit“ ist für mich bedeutend. Auch wenn es eine gute Möglichkeit darstellt, den Lerngegenstand auf eine uns natürliche - eine uns im Alltag vertraute - Weise zu begegnen, so spannen die Konzeptionen Schule und auch die Lernwerkstattarbeit zusätzlich einen bildungsrechtlichen Rahmen. In diesem fließen neben selbstbestimmenden Projekten, in denen Lernende auf eigenverantwortlicher und begleiteter Basis arbeiten, auch lehrende Aspekte mit ein, bei denen Expert*innen auf ihren unterschiedlichen Gebieten vermitteln (sei es in naturwissenschaftlichen, rhetorischen, sozial-wertevermittelnden etc. Bereichen). Das Zusammenspiel von Lernen und Lehren wird durch das gemeinsame Dokumentieren, Auswerten, Analysieren und Reflektieren erzeugt, was im Alltag so nicht stattfindet, was Schule als Bildungseinrichtung und Lernwerkstattarbeit legitimiert. So erfolgte mein Lernprozess schließlich nicht nur über Gespräche, sondern auch (wenn nicht sogar vor allem) über das gemeinsame Analysieren sowie Reflektieren meiner und der anderen Ergebnisse, Erfahrungen, Arbeitsweisen (in der Reflexionsrunde/4. Arbeitsphase) und meiner schriftlichen Auseinandersetzung (Studiennachweis: schriftliche Reflexion der Lernerfahrung) mit dem Thema. Erst ab diesem Schritt begann bei mir der Lernprozess an Geschwindigkeit und Bedeutung zuzunehmen. Nicht nur seine eigenen Arbeitsweisen, Erfahrungen etc. zu reflektieren, sondern auch die der anderen und diese miteinander zu vergleichen und in Beziehung zu setzen, war sehr spannungsvoll und bereichernd. Ebenso später weitere Literatur hinzuzuziehen gab mir viel Aufschluss über meinen Lernprozess.

So habe ich ebenfalls festgestellt, dass man sich durchaus nicht nur in einer Rolle wiederfinden kann. Der Grat zwischen Lernbegleitung und Lernende* ${ }^{*}$ kann durchaus sehr schmal sein, wenn man sich nicht nur konkret auf seinen eigenen Prozess bezieht.

Denkbar ist aber auch eine Überlappung der beiden Rollen, wenn man das Lernbegleiten als Lernziel hat.

„Metakognition bezeichnet die Auswahl der besten Vorgehensweise bei einer Lernaufgabe. Lernende mit guten metakognitiven Fertigkeiten setzen sich Ziele, organisieren ihre Aktivitäten, wählen zwischen verschiedenen Vorgehensweisen beim Lernen aus und wechseln die Strategien, wenn notwendig. "

(WoOLFOLK, 2014, 317) 
Metakognitive Fähigkeiten und Fertigkeiten können bei dem Prinzip der Lernwerkstattarbeit gefördert werden, was man am eigenen Leib gespürt hat. Ich habe mehr über die Art und Weise des eigenen Lernens erfahren und versuche diese in Zukunft bewusst anzuwenden und umzusetzen. Zum Beispiel, dass ich in Lerngruppen effektive Ergebnisse erzielen könnte oder dass ich mir bei einem Lernprozess häufiger das Ziel meines eigentlichen Lernens vor Augen führen sollte, damit ich meine Arbeitsweise und Strategie gegebenenfalls nochmal anpassen kann. Der Aspekt des Trainings oder des Bewusstwerdens der eigenen Metakognition ist auch für Schüler*innen ein wichtiges Thema. So ist die Individualität der Metakognition von Menschen unter anderem auf ihre unterschiedlichen Entwicklungsprozesse zurückzuführen. Kinder beginnen erst im Alter von 5 bis 7 Jahren mit der Entwicklung von metakognitiven Fähigkeiten (vgl. Woolfolk, 2014, 317). Die Aufgabe der Lehrer*innen wird also auch sein, ob durch das Arbeiten in einer Lernwerkstatt oder das Arbeiten im regulären Unterricht, bei den Schüler*innen metakognitive Fähigkeiten zu fördern, damit die Schüler*innen mehr über ihr eigenes Lernverhalten und den eigenen Arbeitsprozess verstehen und so zu besseren Ergebnissen kommen können. Dass Selbstreflexion förderlich sein kann, führt auch Anita Woolfolk $(2014,318)$ auf, indem sie von einem Lehrer berichtet, der bewusst durch gezielte Fragen seine Schüler*innen zur Selbstreflexion anregt. Dies führte zu verbesserten Leistungen und zur Entwicklung metakognitiven Wissens. Aber nicht nur diese Erkenntnisse konnte ich gewinnen. Auch bin ich der Meinung, dass das Projekt ohne den respektvollen und emphatischen Umgang miteinander nicht in der Form umsetzbar gewesen wäre. Hätte sich jeder nur für sein Thema interessiert und hätte nicht den Raum für das Auseinandersetzen mit den anderen Themen zugelassen, so hätte man als Lernbegleitung erschwerten $\mathrm{Zu}$ gang zu den Lernenden gehabt und als Lernende* $r$ wäre die eigene Selbstreflexion weniger zustande gekommen. Daher schließe ich auch daraus, dass ein gesundes Sozialleben ein wichtiger Faktor ist, um sich im Arbeitsprozess kognitiv weiterzuentwickeln, aber auch um Freundschaften aufzubauen und zu halten. Weswegen ich dies in meinem späteren Wirken ebenfalls fördern möchte. Dies kann durchaus mittels der Lernwerkstattarbeit geschehen, wo die Schüler*innen in der Lage sind mit Klassenkamerad*innen ins Gespräch zu kommen, verschiedene Gedanken auszutauschen und sich so weiterzuentwickeln oder gemeinsam als Gruppe (erfolgreich) an einem Projekt zu arbeiten. 


\section{Fazit}

In Bezug zur Lehre in einer Hochschullernwerkstatt kann ich folgendes Resümee ziehen: Nicht nur durch meine direkte Konfrontation als studentische Mitarbeiterin der Hochschullernwerkstatt der Martin-Luther-Universität Halle-Wittenberg sehe ich die Potenziale, die sie für die Hochschullehre von Lehramtsstudierenden innehat. Auch das Seminar gab mir weiteren Aufschluss darüber, wie sehr es den Studierenden bei der Professionalisierung des Berufs Lehrer*in nützen kann. Das Konzept der Lernwerkstatt(arbeit) ist sehr komplex. Alleine schon die Bestimmung einer guten oder schlechten Lernbegleitung kann halbe Bücher füllen. Daher bietet sich eine Hochschullernwerkstatt in einer Universität sehr gut an, so können einerseits die Studierenden handlungsorientiert arbeiten und die kooperative und forschende Lernform am eigenen Erfahrungsbild und Interessenhorizonts durchdringen und verstehen. Andererseits ist das Prinzip der Lernbegleitung (in der Hochschullernwerkstatt) ein wichtiger Aspekt, der ein fester und kein wahlobligatorischer Bestandteil des Curriculums werden sollte. Durch ihn könnte der gesamte und nicht nur ein interessierter Studierendenkreis den Weg zu einer*einem reflektierten Lernbegleiter*in finden und womöglich auch einen besseren Zugang zu diesem offenen Lehr- und Lernkonzept entwickeln. Man muss sich darauf einlassen können, sich mit ihnen kritisch auseinandersetzen, ihnen Raum zur Entfaltung geben, das Konzept direkt am eigenen Leibe erfahren zu können und nicht nur theoretisch oder ansatzweise praktisch abzuhandeln, wie es doch häufig der Fall ist. Dadurch könnten mehr zukünftige Lehrer*innen die Potenziale dieser Form entdecken. Allerdings sollte man auch den großen sachlichen Input betrachten, der in einer Universität vermittelt werden muss. So ergeben sich Vorlesungen, die sehr informativ gehalten sind, um schließlich die Fachkompetenz zu legitimieren. Dies sollte auch weiterhin ein fester Bestandteil bleiben. Allerdings darf meiner Meinung nach dabei diese Vereinigung von Praxis und Theorie, die mittels Lernwerkstattarbeit in einer Hochschullernwerkstatt entsteht, nicht außer Acht gelassen werden.

\section{Literatur}

Dewey, John (2011): Demokratie und Erziehung. Eine Einleitung in die philosophische Pädagogik. Weinheim und Basel: Beltz.

PIetsch, Susanne (2010): Begleiten und begleitet werden. Praxisnahe Fallarbeit. Kassel: Kassel University Press.

Woolfolk, Anita (2014): Pädagogische Psychologie. Hallbergmoos: Pearson Deutschland. 